\title{
Litoestratigrafía fotogeológica del volcán Xictle y su importancia en el registro geológico del sur de la cuenca de México
}

\author{
J. Uribe Luna y V. M. Sotelo Hernández \\ Facultad de Ingeniería, UNAM \\ EmailJesus.Uribe@gecitsla.ge.com
}

(recibido: agosto, 1997; aceptado: marzo, 1998)

\section{Resumen}

El volcanismo reciente en el sur de la cuenca de México está representado por el derrame del volcán Xictle, el cual, dada su expresión superficial, puede ser estudiado con herramientas de sensores remotos como la fotografía aérea. A través de la fotogeología se ha determinado que el derrame está constituido por tres derrames sucesivos de basalto, emitido por tres diferentes ventanas o cráteres volcánicos con las mismas texturas y estructuras que suponen una evolución a partir de una misma cámara magmática. Las rocas son agrupadas y consideradas dentro de la unidad que aquí se denomina "basalto Xictle" por su nomenclatura litoestratigráfica, lo cual en un futuro permitirá definir las relaciones espaciales y temporales con otras unidades volcánicas en el sur de la cuenca de México, como la definida "formación Chichinautzin".

\section{Abstract}

Recent volcanism within Mexico's basin is been represented by Xictle volcano which can be studied by remote sensors as aerial photographs. The photogeology of that volcano shows three basalt layered rocks erupted by three volcanic craters through a single magmatic camera. With the same texture and structure geologic features, this igneous rock could be named "basalto Xictle", by its litho stratigraphic nomenclature that will allow to define spatial and time relationship with others igneous rock like the Pleistocene igneous formation "formación Chichinautzin" at southern Mexico City.

\section{Introducción}

El propósito de este trabajo es la delimitación espacial y la consideración de atributos obtenidos por diversos autores para la definición de una unidad litoestratigráfica que permita entender la evolución volcánica de la sierra Chichinautzin. Los datos disponibles de estratigrafía (De Cserna Zoltán, et al, 1988; Delgado Granados, et al, 1994), paleomagnetismo (Centeno Elena, et al, 1986), arqueología y vegetación (Rzedowski, 1954), composición litológica (Badilla Cruz, 1977; Martín del Pozo, 1982) y edades radiométricas (Urrutia Fucugauchi, 1996) de la zona conocida como "pedregal de San Ángel" han contribuido a la caracterización de un evento volcánico reciente dentro de la cuenca de México (tabla 1). El volcán Xictle ha sido definido como un evento de origen monogenético único, ("Xictli", denominado en otros trabajos "Xitle"), lo que ha llevado a una generalización de la evolución volcánica, particularmente en el extremo norte de la sierra Chichinautzin.

Mediante el uso de fotografía aérea y sistemas de información geográfica es posible obtener un mayor número de detalles y atributos físicos (Fig. 1) que ayuden a definir con más precisión una unidad litoestratigráfica, trabajo básico para comprender la evolución en tiempo y espacio del volcanismo reciente.

El Xictle es un claro ejemplo de las manifestaciones volcánicas dentro de la cuenca de México (Fig. 2); entender las

Tabla 1. Atributos de las principales unidades fotogeológicas interpretadas

\begin{tabular}{ccccccc}
\hline No. & Longitud (W) & Latitud $(\mathrm{N})$ & Nombre & Roca & Altura $(\mathrm{m})$ & Diámetro $(\mathrm{m})$ \\
\hline 1 & $-99^{\circ} 13^{\prime} 20^{\prime \prime}$ & $19^{\circ} 14^{\prime} 40^{\prime \prime}$ & Xictle & Basalto & 120 & 700 \\
2 & $-99^{\circ} 13^{\prime} 36^{\prime \prime}$ & $19^{\circ} 14^{\prime} 35^{\prime \prime}$ & Xictontle & Basalto & 40 & 440 \\
3 & $-99^{\circ} 13^{\prime} 05^{\prime \prime}$ & $19^{\circ} 14^{\prime} 34^{\prime \prime}$ & Sin nombre & Basalto & 25 & 260 \\
4 & $-99^{\circ} 14^{\prime} 00^{\prime \prime}$ & $19^{\circ} 15^{\prime} 50^{\prime \prime}$ & Cuilotepec & Basalto & 10 & 100 \\
5 & $-99^{\circ} 12^{\prime} 46^{\prime \prime}$ & $19^{\circ} 14^{\prime} 29^{\prime \prime}$ & Cuautzontle & Andesita & 80 & 760 \\
\hline
\end{tabular}




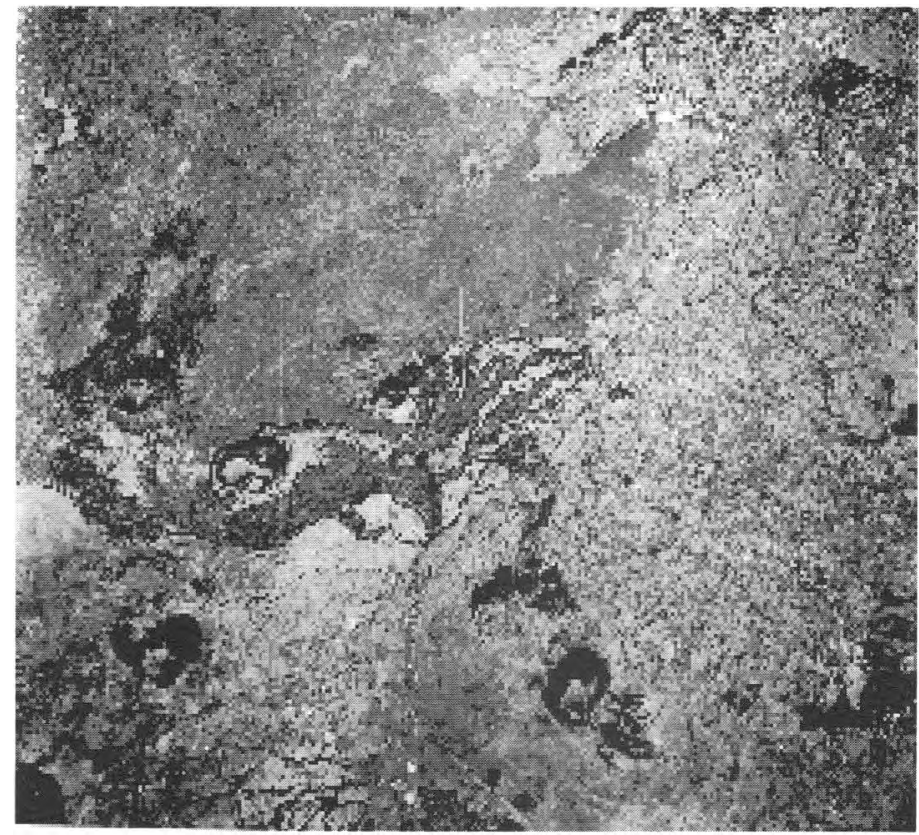

Figura 1. Fotografia aérea de 1970 de las unidades litoestratigráficas del volcán Xictle geológicamente fotointerpretada para su representación digital.

características estratigráficas de tales eventos volcánicos nos permitiría elaborar modelos de evolución del volcanismo en dicha cuenca.

\section{Litoestratigrafía del volcán Xictle}

Una unidad litoestratigráfica está definida con base en el carácter litológico de los materiales de la superficie terrestre (Hedberg, 1980), atributo físico que permite delimitar y separar espacialmente los materiales de la superficie terrestre. Las rocas del Xictle son una porción del conjunto de rocas que constituyen a la sierra Chichinautzin, la cual está constituida a su vez, en general, por antiguos derrames lávicos con estucturas de tipo "pahoehoe", de superficies cordadas, así como de bloques tipo "aa" y estructuras constructivas de escudo-volcanes, estratovolcanes y conos cineríticos (Martín del Pozo, 1982).

El conjunto de rocas de dicha sierra ha sido denominado "serie basáltica Chichinautzin" (Mooser, 1956; Gunn y Mooser, 1970), "grupo Chichinautzin" (Fries, 1960) y posteriormente, "formación Chichinautzin" (Schlaepfer, 1968). Sin embargo, la definición es aún incompleta porque no considera las características y atributos de las diferentes unidades litológicas de las estructuras volcánicas individuales. La importancia de organizar los cuerpos de roca volcánica en dicha sierra en función de su carácter litológico radica primero en definir y delimitar las particularidades de la misma y después en la definición de una formación litoestratigráfica formal para tener un mejor

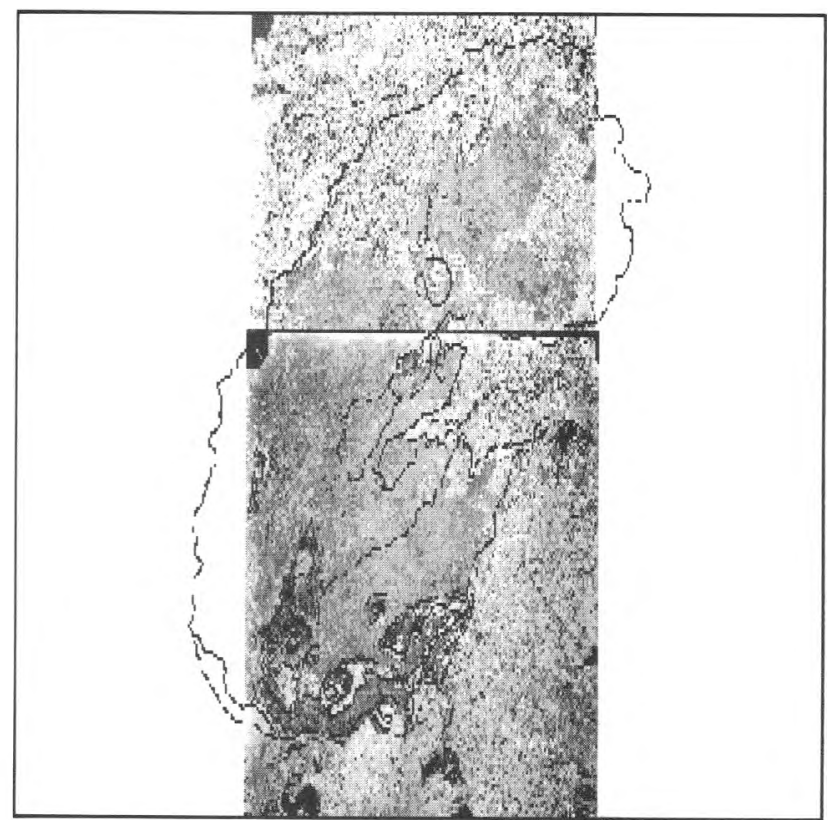

Figura 2. Unidades fotointerpretadas de un par estereóscopico del volcán Xictle y volcanes adyacentes al sur de la ciudad de México.

control estratigráfico y por tanto de la evolución volcánica; ello permitirá tener en el futuro un modelo evolutivo geológico (Mooser, 1956) de la cuenca de México.

Las rocas volcánicas pueden ser consideradas como burdamente estratificadas y es posible establecer la posición relativa de aquellas mediante métodos indirectos, paticularmente con la interpretación de fotografías aéreas (Fig. 2). En trabajos anteriores se ha definido que la cuenca de México es el resultado del cierre de una antigua cuenca exorreica por el volcanismo pliocénico-pleistocénico; sin embargo, la presencia de depósitos volcánico-sedimentarios de edad anterior al Plioceno, de más de $2000 \mathrm{~m}$ de espesor dentro de la cuenca y la ausencia de éstos en el borde sur de la misma, sugiere una evolución de cuenca endorreica estructural al menos desde el Mioceno Tardío (Oviedo de León, 1970).

\section{Definición de la unidad litoestratigráfica}

La unidad litoestratigráfica propuesta aquí como "basalto Xictle" está constituida por tres derrames sucesivos de basalto de olivino, de estructura interna y derrame volcánico denso en la base y vesicular en la cima, por la presencia de una fase gaseosa durante el derrame de estructura superficial pahoehoe y en bloques. Tiene una extensión superficial de $70.4 \mathrm{~km}^{2}$ y un espesor promedio de $16 \mathrm{~m}$, con una estimación volumétrica de $0.4 \mathrm{~km}^{3}$.

El uso de fotografías aéreas (Fig. 1) es la alternativa más práctica y económica para definir las características de unidades litoestratigráficas con base en los criterios de tono, 
textura, tamaño y forma, entre otros. Dicha herramienta y la integración de una base de datos en un sistema de información geográfica permiten obtener un mayor número de detalles de las relaciones estructurales y estratigráficas en tiempo y espacio.

Las rocas del Xictle sobreyacen a rocas volcánicas piroclásticas de composición andesítica de la sierra de Las Cruces, de edad pliocénica y a las estructuras volcánicas erosionadas de los cerros Zacatépetl y parque nacional Bosque del Pedregal (Fig. 2), compuestas de andesita porfídica y brecha volcánica de edad pliocénica; asimismo, sobreyacen a otras estructuras claramente identificables como son las cónicas truncadas y abiertas de los volcanes Cuilotepec, constituido por toba de lapilli basáltico y Cuautzontle, formado por ande. sita de estructura superficial de derrame en bloques.

El evento volcánico más antiguo del Xictle está representado por el derrame de un volcán sin nombre, localizado $1 \mathrm{~km}$ del sureste del Xictle; fotogeológicamente tiene tono gris obscuro, textura fina, geoforma cónica, dirección de flujo $\mathrm{N} 15^{\circ} \mathrm{E}$ con extensión hacia el parque nacional Fuentes Brotantes, espesor promedio de $5 \mathrm{~m}$ y superficie de $11.5 \mathrm{~km}^{2}$. Este se encuentra sobrepuesto por el derrame del Xictle, el cual tiene tono gris claro, textura fina, geoforma cónica, dirección de flujo $\mathrm{N} 48^{\circ} \mathrm{E}$ que se extiende hacia la zona de San Ángel, Santo Domingo, Copilco, Santa Úrsula y San Fernando, espesor promedio de $16 \mathrm{~m}$, y superficie de $55.4 \mathrm{~km}^{2}$.

Dentro de un banco de la material de la avenida Dalias, en la ciudad universitaria (Fig. 3), se registró un espesor de $36 \mathrm{~m}$, el cual presenta dos superficies de enfriamiento en la sección del corte de roca; éstas son identificables por la presencia de superficies cordadas que muestran claramente la presencia de varios eventos de derrame de la unidad. Dicho banco se encuentra a su vez sobrepuesto por el derrame del volcán Xictontle, de tono

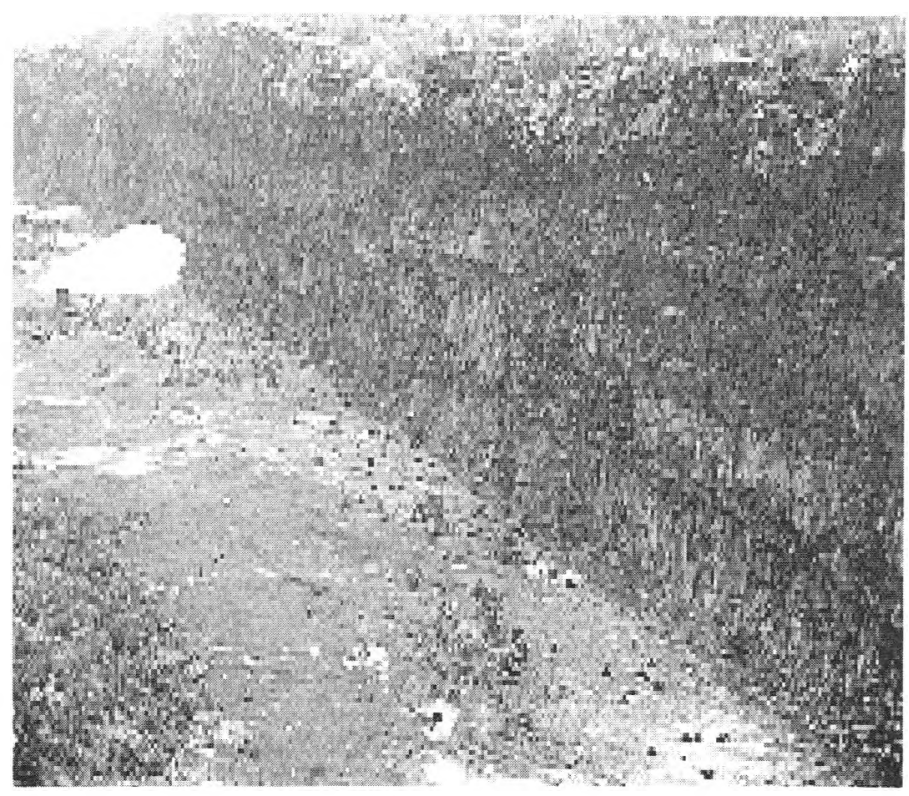

Figura 3. Unidad litoestratigráfica del volcán Xictle en el corte del banco de material de avenida Dalias, 1991.

gris y textura granular fina, estructura cónica con dirección de flujo de derrame de $S 70^{\circ} \mathrm{E}$ que se extendió hacia al sur y sureste, bordea al Xictle y llega a cubrir parcialmente al Cuautzontle, con un espesor promedio de $5 \mathrm{~m}$ y superficie de $3.4 \mathrm{~km}^{2}$ (tabla 2).

En la literatura y fuentes bibliográficas se encuentran datos de fechamientos por radiocarbono con un valor definido en 2,422 250 años antes del presente (AP); en estudios recientes se ha definido que las edades documentadas varían entre $4,790 \pm 80$ y $1,430 \pm 200$ años AP (Urrutia Fucugauchi, 1996). Es muy probable que parte del amplio rango de edad se deba al muestreo sobre los diferentes derrames volcánicos.

Tabla 2. Atributos geológicos de las unidades fotogeológicas interpretadas

\begin{tabular}{cccc}
\hline No. & Sitio & Espesor $(\mathrm{m})$ & Dirección de flujo \\
\hline 1 & Parque Nacional Bosque del & & $\mathrm{N} 15^{\circ} \mathrm{E}$ \\
2 & Pedregal & 1 & $\mathrm{~N} 15^{\circ} \mathrm{E}$ \\
& Banco de material & & \\
3 & CU., Av. Dalias. & 36 & - \\
4 & Presa Anzaldo & 1.5 & $\mathrm{~N} 5^{\circ} \mathrm{W}$ \\
5 & Periférico sur & 8 & $\mathrm{~N} 40^{\circ} \mathrm{E}$ \\
6 & Arroyo Chichicuautita & 6 & - \\
7 & Arroyo Chichicuautita & 5 & $\mathrm{~N} 53^{\circ} \mathrm{E}$ \\
8 & Lomas Padierna & 6 & $\mathrm{~N} 48^{\circ} \mathrm{E}$ \\
9 & Cráter del volcán Xictle & 2 & $\mathrm{~N} 62^{\circ} \mathrm{E}$ \\
10 & Cuicuilco & 3 & - \\
11 & Espacio escultórico CU. & 3 & - \\
12 & Pedregal de San Francisco & 7.6 & 8 \\
13 & Av. Universidad y M A. de Quevedo & 18 & \\
\hline
\end{tabular}


Los fechamientos radiométricos de restos de madera encontrados por debajo del derrame indican edades de 2,422 años AP, pero la madera pudo haber sido quemada antes de ser cubierta por el derrame, arrojando así un valor erróneo de edad; por otra parte, la relación por sobreposición con los sitios arqueológicos de Tizapán, Copilco y en especial Cuicuilco, que prevalecieron hasta el final del periodo preclásico (100 años AP), sugiere un valor conservador de 2000 años AP para el derrame del Xictle (Fig.4).

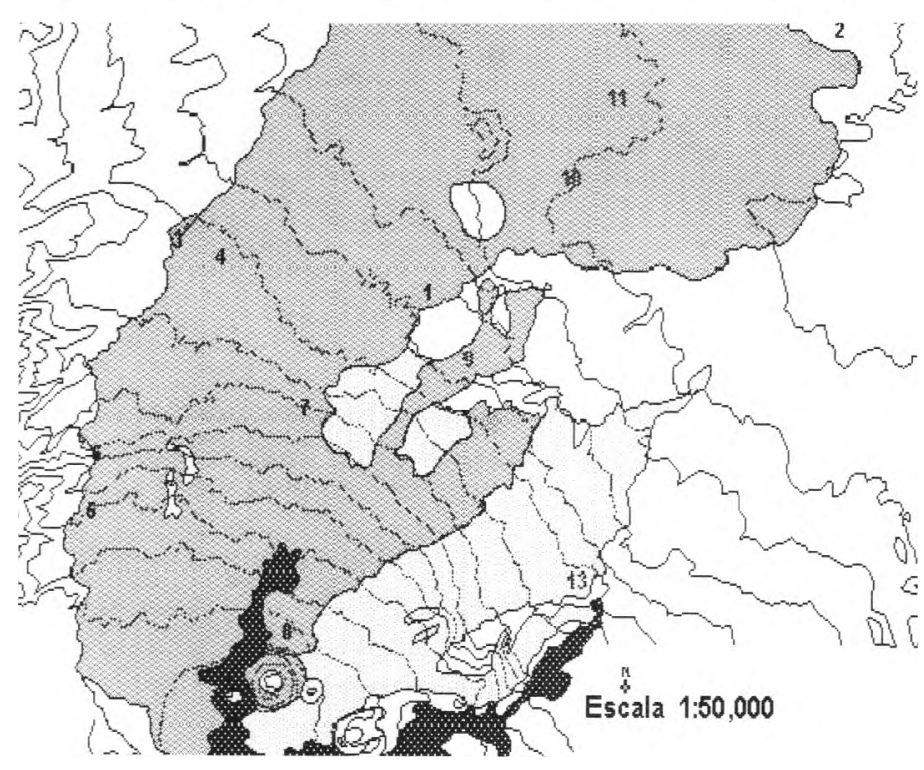

Figura 4. Mapa de localización de los sitios de la tabla 2 de la unidad "basalto Xictle". En gris claro, derrame del volcán Xictontle, el más antiguo; en gris obscuro, derrame del volcán Xictle; en negro, derrame del volcán sin nombre, el más joven, al oeste del Xictle

Si se considera la definición de una unidad litoestratigráfica independiente del atributo de tiempo geológico, el conjunto de rocas del Xictle puede ser referido con el nombre compuesto "basalto Xictle" que considera el caráter litológico de la unidad y el nombre geográfico de un elemento natural que además está de acuerdo con las definiciones de la nomenclatura estratigráfica (Comisión Americana de Nomenclatura Estratigráfica, 1970) y tiene el equivalente de capa en la clasificación de rocas sedimentarias (Código Estratigráfico Norteamericano, 1983).

\section{Conclusiones}

Las rocas del derrame del volcán Xictle deben ser referidas ya sea por su carácter litológico o bien por su posición estratrigráfica con respecto a rocas más antiguas; el derrame lávico del Xictle se propone como unidad "basalto Xictle" y no como pedregal de San Ángel, nombre impreciso para una estructura volcánica claramente identificable y geológicamente documentable en el registro volcánico holocénico dentro de la cuenca de México.

La fotogeología permite obtener mayor detalle para definir a la unidad litoestratigráfica como un conjunto de rocas emitidas a partir de una misma cámara magmática a través de tres diferentes ventanas o cráteres, en una aparente estructura orientada este-oeste, reflejo de estructuras mayores de expresión regional. Estas estructuras no han sido comprendidas, pero parecen haber jugado un papel importante en la evolución de la cuenca de México, de tipo estructural endorreica.

La edad documentada en otros trabajos, que ha sido obtenida por métodos no inherentes a los cuerpos de roca, así como otros métodos geofísicos (Urrutia Fucugauchi y Martín del Pozo, 1993) y parte del rango de edad obtenido, probablemente corresponde a los diferentes derrames identificados; sin embargo, el atributo de tiempo es independiente de la definición de la unidad litoestratigráfica "basalto Xictle"; las relaciones con otras unidades litoestratigráficas de la sierra Chichinautzin permitirán definir apropiadamente la formación Chichinautzin constituida a su vez por unidades litoestratigráficas definidas tanto por sus atributos como por su cartografiabilidad documentada y referida en otros trabajos.

\section{Referencias}

Badilla Cruz R.R.(1977). Estudio petrológico de la lava de la parte noreste del pedregal de San Ángel. Sociedad Geológica Mexicana, México.

Centeno E., Urrutia Fucugauchi J. y Herrero B.E. (1986). Diferenciación y caracterización de flujos de lava por medio de sus propiedades magnéticas-pedregal de San Ángel, México, en: Reunión Anual. Union Geofísica Mexicana. GEOS..

Comisión Americana de Nomenclatura Estatigráfica. Ed. (1983). Código Estratigráfico Norteamericano.

Comisión Americana de Nomenclatura Estratigráfica. Ed. (1970). Código de Nomenclatura Estratigráfica.

De Cserna Z., et al (1988). Estructura geológica, gravimetría, sismicidad y relaciones tectónicas regionales de la cuenca de México. Instituto de Geología, UNAM, México.

Delgado Granados H., et al (1991). Eruptive History of Xitle Volcano (southern Mexico city): volcanic risk implications. GEOS, 84-85.

Fries C. (1960). Geologia del estado de Morelos y de partes adyacentes de México y Guerrero, región centromeridional de México. Instituto de Geología, UNAM, México. 
Gunn B. y Mooser F. (1970). Geochemistry of the volcanoes of Central Mexico. Volcanologique, Vol. 34, 577-616.

Hedberg H. (1980). Guía estratigráfica internacional. Reverté.

Martín del Pozo A. (1982). Monogenetic Vulcanism in Sierra Chichinautzin, Mexico. Volcanology, Vol.45, 9.24.

Mooser F. (1956). Los ciclos del volcanismo que formaron la cuenca de México, en: XX Congreso Geológico Nacional, Vol. C-6, pp. 337-348.

Oviedo de León A. (1970). El conglomerado Texcoco y el posible origen de la cuenca de México. Instituto Mexicano del Petróleo, 5-20.
Rzedowski J. (1954). Vegetación del pedregal de San Ángel, en: Anales de la Escuela Nacional de Ciencias Biológicas. No. 8, pp. 59-129.

Schlaepfer C. (1968). Hoja Distrito Federal y Estados de México y Morelos, Esc. 1:100,000. Instituto de Geología, UNAM.

Urrutia Fucugauchi J. (1996). Comentarios sobre la edad del campo volcánico pedregal de San Ángel, cuenca de México-fechamientos por radiocarbono. GEOS, Vol.16, 96-98.

Urrutia Fucugauchi J. y Martín del Pozo, P. (1993). Implicaciones de los datos paleomagnéticos sobre la edad de la sierra Chichinautzin, cuenca de México. Geofisica Internacional, Vol.32, 523-533. 